\title{
Assessment of Knowledge, Attitude and Adherence to Treatment in Patients With Heart Disease in Iran
}

\author{
Masoud Khodaveisi', Shahla Fakhrazizi', Nahid Mohammadi', Leili Tapak² \\ ${ }^{1}$ Department of Community Health Nursing, School of Nursing and Midwifery, Hamedan University of Medical Sciences, \\ Hamedan, Iran \\ ${ }^{2}$ Department of Epidemiology, School of Public Health, Hamedan University of Medical Sciences, Hamedan, Iran
}

Corresponding Author: Leili Tapak, PhD in Biostatistics, Assistant Professor, Department of Epidemiology, School of Public Health, Hamedan University of Medical Sciences, Hamedan, Iran. Tel: +98-8138380090, Email: I.tapak@umsha.ac.ir

Received May 17, 2019 ; Accepted October 26, 2019; Online Published November 26, 2019

\begin{abstract}
Introduction: Adherence to treatment is essential in heart disease. This study was conducted to evaluate the nutritional awareness of hypertensive patients and their dietary intake in heart disease.

Methods: This is a descriptive-analytical study that was performed on hypertensive patients referred to Farshchian Cardiovascular Clinic in Iran, using the available random sampling method. Data collection tools included demographic information questionnaire and three questionnaires of nutritional awareness, illness perception, and dietary follow-up of patients with hypertension. Data were analyzed using SPSS software version 16 and descriptive statistics.

Results: The mean score of knowledge was $4.73 \pm 1.55$. Fifty-eight percent of patients were unaware of high-fat diet, $56 \%$ of them did not eat red meat and $63 \%$ of them did not eat sweets. The mean score of perception was $29.58 \pm 3.94$ of which $38 \%$ of the patients were uncontrollable and $43 \%$ strongly opposed their illnesses which could lead to premature death and $56 \%$ were against dietary restriction. Conclusion: There is a need for interventions to improve the knowledge and attitude of patients with heart disease.

Keywords: Knowledge, Attitude, Heart Disease, Adherence Treatment
\end{abstract}

Citation: Khodaveisi M, Fakhrazizi S, Mohammadi N, Tapak L. Assessment of knowledge, attitude and adherence to treatment in patients with heart disease in Iran. Int J Travel Med Glob Health. 2019;7(4):142-146. doi:10.15171/ijtmgh.2019.29.

\section{Introduction}

Coronary artery disease is the main cause of death and disability in industrialized countries and also the cause for $30 \%$ of all deaths. Hypertension is one of the common, chronic, recurrent diseases that sets the context for many diseases. Hypertension accounts for $5 \%$ of the world's disease burden, which affects more than a quarter of the world's adult population. It is projected that by 2025 the disease rate increases by $24 \%$ in developed countries and $80 \%$ in developing countries. ${ }^{1}$

This disease is very critical given its high prevalence, yet the point doubling its significance is that hypertension is an uncontrolled disease. Studies have shown that the role of nutrition in controlling this disease is undeniable, unfortunately there is a much evidence in contrast, and most patients do not actually heed dietary guidelines and only half of the patients have accepted following proper diet as part of the treatment. Following hypertension, it is important to control high blood pressure to minimize the probability of complications. ${ }^{2,3}$ It appears that the root of these behavior and beliefs, inaccurate information, and poor awareness of the nature of the disease and nutrition are related. As one of the reasons for not controlling hypertension, poor nutritional awareness of patients has been reported and lack of information in these patients is considered an important issue. ${ }^{4,5}$ Although it has been determined that awareness without other factors may be unable to modify behavior, it can be a determinant of one's dietary behavior. ${ }^{6}$ Indeed, patient's knowledge is an important factor in accepting treatment and controlling these diseases.

A wide range of health care providers provide their services in the health system, yet nurses shape the base of the health care delivery system and have a critical role in coordinating and delivering health care. As nurses work with families in a variety of contexts such as clinics and schools, the significant role of community-based nursing is to reduce health risks, improve the health of various populations, and provide services to families. ${ }^{7}$ 
Hence, given the importance and effect of nutritional behavioral and awareness changes in health promotion and disease control and the use of care, the study was conducted to evaluate nutritional awareness of hypertensive patients and their dietary intake in patients admitted to Farshchian cardiovascular clinic in Hamadan, Iran in 2017.

\section{Methods}

Study Design and Participants

The present descriptive study was conducted in Farshchian cardiovascular clinic in 2017. In the study, 100 hypertensive patients and the inclusion criteria were selected using a convenient random sampling method. The sample size was calculated according to Kamran et al's ${ }^{8}$ study.

\section{Inclusion and Exclusion Criteria}

Inclusion criteria were 30-60 years of age, mild to moderate hypertension approved by a physician, having at least 1-year history of hypertension, and having no known underlying disease in severe and critical stage (such as cancer, metastatic, and renal failure).

\section{Sample, Sampling Strategy, and Sample Size}

The data collection tool was a four-part questionnaire, including (1) Demographic characteristics questions, (2) Nutrition awareness questions, including 10 true-false questions with 1 scored for the correct answer and zero to "I do not know" and the wrong answer. (3) Hypertension perception questions were 14 questions in 2 domains based on 4-option Likert attitude scale (completely agree, agree, disagree, and completely disagree), where corresponds to response, scores 1 to 4 were given, with scores range 14-56. (4) Diet follow-up questions were 5 questions based on a 5-option scale (always, often, sometimes, rarely, never) with scores on these questions ranging from 1 to 5 and the total score ranging from 5 to 25 points. Obtaining points for each aspect summed up the scores for each of the following questions, with scores on illness perceptions ranging from 14 to 56 and nutritional follow-up questions from 5 to 25 and nutritional awareness questions 0 to 10 . Then for ranking, the total score of each aspect is calculated based on 100 and the mean scores are set at three levels of desirable (mean score above 75), average (mean score 50-75) and unfavorable (mean score less than 50). In Kamran et al's study, ${ }^{8}$ Cronbach's alpha, split-half, and Richardson coder were employed to examine the reliability, and the validity of the questionnaire was assessed by content and construct validity. The results of Spearman Brown coefficient, Cronbach alpha, Gutman and Coder Richardson in the questionnaire were satisfactory, which showed satisfactory reliability of the questionnaire. Questionnaire questions were highly correlated with the total score and internal consistency analysis showed that the questionnaire questions had acceptable reliability. In factor analysis, all questionnaire questions had acceptable factor loadings and based on the factor analysis, all questionnaire questions were significant and had appropriate construct validity. Moreover, this questionnaire was approved by professors with a validity coefficient which was above 0.90 . Cronbach's alpha for all aspects was above 0.80 . In the study of Kamran et $a{ }^{8}{ }^{8}$ both validity and reliability of this questionnaire were confirmed by colleagues.

\section{Outcome}

After determining the number of participants among those admitted to Farshchian cardiovascular clinic in Hamadan, Iran those with the inclusion criteria were selected after obtaining their consent, as the researcher fully explained the research process to them. To avoid the effect of some dummy variables, their effects were controlled by modeling in data analysis. Then, the questionnaires were filled out by the individuals. The demographic questionnaire was completed and then the questionnaires of nutritional knowledge, illness perception, and dietary follow-up were completed in hypertensive patients.

\section{Statistics}

Descriptive statistics was used to analyze the data. Data were then analyzed using SPSS version 16 software. Descriptive statistics, such as mean and standard deviation (for quantitative variables such as age, duration of illness), as well as indices of number and percentage were used to report other cases.

\section{Results}

One-hundred patient were included in this study. According to the results, the mean score of knowledge was $4.73 \pm 1.55$ and according to the questionnaire, $58 \%$ of patients did not have the necessary knowledge about high-fat diet and 56\% did not know that eating red meat was effective in raising blood pressure (Table 1).

The mean score in the perception domain according to the results of the questionnaire was $29.58 \pm 3.94$. Thirty-eight percent stated that the disease was behaviorally uncontrollable. Then, $43 \%$ of patients strongly disagreed that it could cause premature death and $56 \%$ did not believe there was a need to restrict salt and fat intake, and only $12 \%$ believed the disease could be a threat to their health (Table 2).

The mean score according to the questionnaire results was $9.45 \pm 2.27$ in the diet follow-up domain and $46 \%$ of the patients stated that they never follow the recommended diet when they are at home, while only $2 \%$ of hypertensive patients adhere strictly to their disease diet at home. Moreover, $29 \%$ of the patients reported that they did not adhere to their diet when in the society and outdoors. According to these results, one can conclude that nutritional awareness, disease perception, and dietary follow-up are impaired in hypertensive patients and need follow-up and remedial action (Table 3 ).

\section{Discussion}

The current study which examines nutritional awareness, illness perception, and dietary follow-up in hypertensive patients showed that those who were more aware and had an understanding of their disease and were more likely to adhere to the treatment regimen were less likely to develop complications. Moreover, patients who believe their health status is under their personal control, feel more responsible 
Table 1. Questions and Percentage of Responses Related to Nutritional Awareness of Patients With Hypertension

\begin{tabular}{|c|c|c|}
\hline Questions & Correct & False \\
\hline Animal fats, such as natural butter increases blood pressure & $42(42 \%)$ & $58(58 \%)$ \\
\hline Bread and whole grains help decrease blood pressure & $40(40 \%)$ & $60(60 \%)$ \\
\hline $\begin{array}{l}\text { The patients with hypertension have to consume at least } 4 \text { to } 5 \text { units of vegetables per day (each unit is equal to one glass of raw } \\
\text { leafy vegetables or one second of cooked vegetable glass). }\end{array}$ & $24(24 \%)$ & $76(76 \%)$ \\
\hline Milk and other low-fat dairy products are harmful to a person with hypertension. & $62(62 \%)$ & $38(38 \%)$ \\
\hline In hypertension, diet is only restricted to salt intake. & $67(67 \%)$ & $33(33 \%)$ \\
\hline Consuming foods like chicken and fish have an effect on raising blood pressure. & $35(35 \%)$ & $65(65 \%)$ \\
\hline A person with hypertension should avoid sweets. & $63(63 \%)$ & $37(37 \%)$ \\
\hline Sweet drinks such as carbonated drinks can have a harmful effect on blood pressure. & $63(63 \%)$ & $37(37 \%)$ \\
\hline
\end{tabular}

Table 2. Questions and Percentage of Responses relevant to Understanding Patients with Hypertension

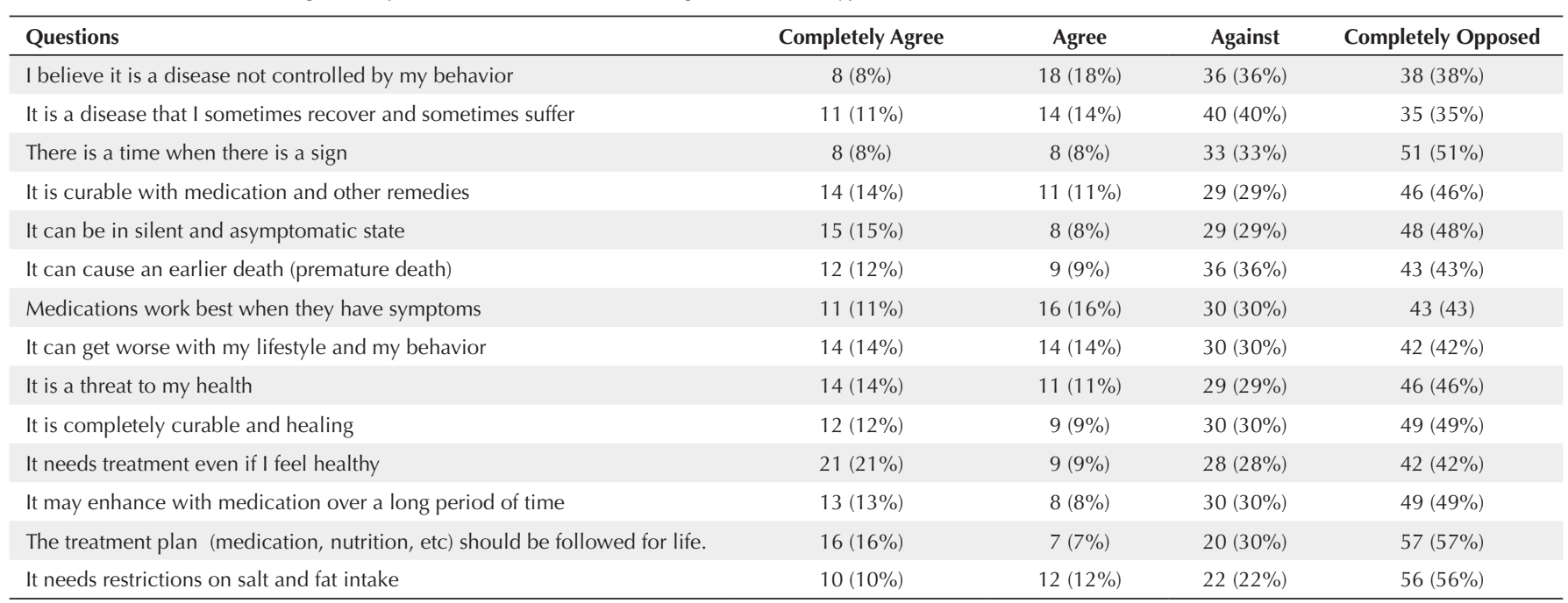

Table 3. Questions and Percentage of Response Relevant to Diet Follow-up of Patients With Hypertension

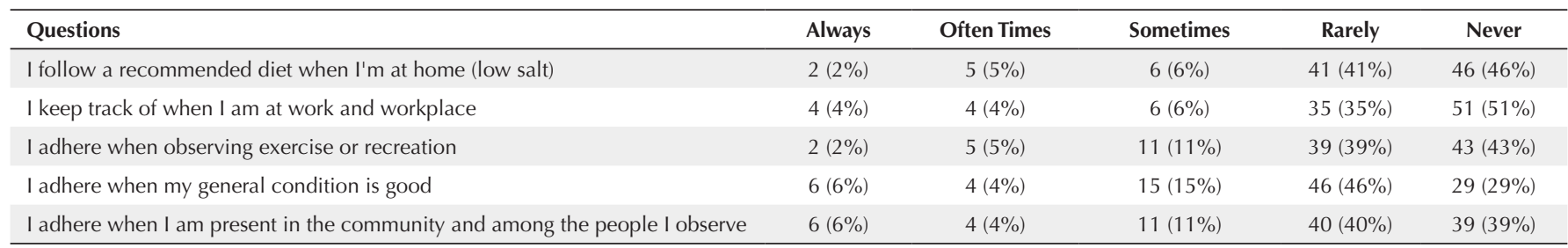

for their health and adhere to treatment. In the examinations by the researcher, few studies were found concerning the perception of the disease in hypertensive patients in Iran, showing the necessity of further studies in this regard.

In the area of awareness, a study that is consistent with the present study is performed by Booth et $\mathrm{al}^{9}$ that addresses patients' need for awareness. The results of their study showed that education (awareness) and follow-up care significantly reduced smoking and reduced salt intake, reducing the risk of heart disease recurrence and mortality.

The results of this study can be used to inform health care managers and service personnel to take necessary measures to increase follow-up training. Adherence to the diet is of great significance in hypertensive patients. This has reduced the number of frequent referrals and hospitalizations to emergencies and clinics, which is both economically and psychologically difficult for patients and families.

Taking this into account, a quasi-experimental study was conducted by Babaei-Sis et al. ${ }^{10}$ According to its results, the mean scores of exercises, weight control, nutrition, and mental health in the experimental group significantly increased after the intervention $(P<0.05)$, but the changes in the control group were not significant $(P<0.05)$. The educational intervention had a significant effect on blood pressure in the experimental group compared to the control group $(P<0.05)$. The results showed that educational intervention was effective in promoting physical activity, improving weight control, nutrition, and mental health in hypertensive patients. Other 
studies that are in line with the present study are those of Cheraghi et $\mathrm{al}^{11}$ and Hosseini et $\mathrm{al}^{2}$ stating with the knowledge gained about diet therapy and the emphasis on diet, one can reduce the complications of the disease.

In understanding domain, the studies conducted by the researcher showed that there are few research studies on the understanding of the disease in Iranian hypertensive patients, suggesting the necessity of further studies in this regard. In the study by Kamran et al, perception was suggested as a determinant in the diet of hypertensive patients. ${ }^{8}$ Perception has been recognized in studies as an important factor in nutritional behavior. These results are justified by behavioral theories as planning for a behavior depends on the outcomes and benefits that are anticipated. In their study, Rajpura and Nayak reported a positive relationship between the perception of illness and positive beliefs about treatment in hypertensive elderly with high treatment adherence. ${ }^{12}$ Moreover, the results of the study by Kang et $\mathrm{al}^{13}$ showed that patients who understand their disease status adhere more to their diet. The results of the study by Saarti et $\mathrm{al}^{14}$ indicated that the mean scores of disease perception in patients with good adherence were higher than those with poorer adherence, yet no significant relationships were observed. In this study, education and home care improved the perception of diet and its relation to the disease in hypertensive patients. Hence, this type of training can be an important step in enhancing patients' understanding of their condition and subsequently improving adherence to specific diet and therapies.

In the dietary follow-up domain, the results suggest that dietary follow-up can have a significant role in improving the process of hypertension and according to the results of the present study, the need for dietary follow-up is quite sensible. Consistent with the present study, Mohammadi et a ${ }^{15}$ performed a follow-up study on the effect of follow-up on blood pressure control in hypertensive patients. Based on their findings, follow-up improved the diet of hypertensive patients. Regarding dietary patterns, there was a significant difference in dietary intake after the intervention.

Among the other studies that have examined and evaluated the need for dietary follow-up, one can cite the ones by Rahimi et $\mathrm{al}^{16}$ or Hacihasanoğlu and Gözüm. ${ }^{17}$ According to the results of this study, one can conclude that by implementing the continuous care model, there is a significant difference in the levels of general and specific aspects of patients' life quality, so that the implementation of this care model is associated with an increase in patients' life quality.

Another study in line with the present study is the one by Hashemi et al, ${ }^{18}$ entitled "Examining the effect of continuous care model on diet adherence of hemodialysis patients." Their study was a controlled clinical trial study conducted on 98 hemodialysis patients in Baqiyatallah and Chamran hospitals in 2013. According to the results, most hemodialysis patients did not have proper dietary compliance. There was a significant relationship between the model of continuous care and diet adherence $(P=0.0001)$. According to the results, patients under the influence of the follow-up care model had better diet adherence by counseling and education, thus it is
Research Highlights

What Is Already Known?

Non-adherence to treatment has led to many deaths and many complications for patients.

\section{What This Study Adds?}

Adherence to treatment is low in cardiac patients in Iran, hence educational interventions are recommended.

recommended that Intensive care units adopt this model to preclude complications and enhance the diet of hemodialysis patients.

\section{Conclusion}

The results of this study showed the need for awareness and increased perception and follow-up of diet in hypertensive patients and non-adherence to pharmacological and nonpharmacological regimens are associated with hypertensive complications, such as stroke. Dietary awareness and understanding of the disease and its increased and effective diet can reduce the complications of hypertension in patients with hypertension.

\section{Authors' Contributions}

Conceptualization, MK, SF; Data curation, NM; Formal analysis, LT, NM; Funding acquisition, MK, SF; Writing original draft, SF, MK; Writing - review and editing, MK, SF, LT, and MN.

\section{Conflict of Interest Disclosures}

The authors declare no conflicts of interest.

\section{Ethical Approval}

This study was approved by Ethics Committee of Hamedan University of Medical Sciences with code IR.UMSHA. REC.1396.41.

\section{Funding/Support}

This study was extracted from the master thesis of community health nursing with code 960129750 .

\section{References}

1. Mills KT, BundyJD, KellyTN, et al. Global disparities of hypertension prevalence and control: a systematic analysis of population-based studies from 90 countries. Circulation. 2016;134(6):441-450. doi:10.1161/circulationaha.115.018912.

2. Hosseini F, Farshidi H, Aghamolaei T, Madani A, Ghanbarnejad A. The impact of an educational intervention based on PRECEDEPROCEED model on lifestyle changes among hypertension patients. Iranian Journal of Health Education and Health Promotion. 2014;2(1):17-26. [Persian].

3. Ndumele CD, Shaykevich S, Williams D, Hicks LS. Disparities in adherence to hypertensive care in urban ambulatory settings. J Health Care Poor Underserved. 2010;21(1):132-143. doi:10.1353/ hpu.0.0259.

4. Sanne S, Muntner P, Kawasaki L, Hyre A, DeSalvo KB. Hypertension knowledge among patients from an urban clinic. Ethn Dis. 2008;18(1):42-47. 
5. Sheahan SL, Fields B. Sodium dietary restriction, knowledge, beliefs, and decision-making behavior of older females. J Am Acad Nurse Pract. 2008;20(4):217-224. doi:10.1111/j.17457599.2008.00307.x.

6. Mohebi S, Azadbakhat L, Feyzi A, Hozoori M, Kamran A, Sharifirad G. Educational needs of women with metabolic syndrome on healthy nutrition in Isfahan: application of health promotion model. Journal of Health. 2013;4(2):165-179. [Persian].

7. Linderholm $M$, Friedrichsen $M$. A desire to be seen: family caregivers' experiences of their caring role in palliative home care. Cancer Nurs. 2010;33(1):28-36. doi:10.1097/ NCC.0b013e3181af4f61.

8. Kamran A, Sharifirad G, Mohebi S, et al. Psychometric assessment of nutritional knowledge, illness perceptions and dietary adherence in hypertensive patients—Ardabil 2013. Health Systems Research. 2013;2014(3):1774-85. [Persian].

9. Booth JN 3rd, Levitan EB, Brown TM, Farkouh ME, Safford MM, Muntner P. Effect of sustaining lifestyle modifications (nonsmoking, weight reduction, physical activity, and mediterranean diet) after healing of myocardial infarction, percutaneous intervention, or coronary bypass (from the REasons for Geographic and Racial Differences in Stroke Study). Am J Cardiol. 2014;113(12):19331940. doi:10.1016/j.amjcard.2014.03.033.

10. Babaei-Sis M, Ranjbaran S, Mahmoodi H, Babazadeh T, Moradi F, Mirzaeian K. The Effect of Educational Intervention of Life Style Modification on Blood Pressure Control in Patients with Hypertension. Journal of Education and Community Health. 2016;3(1):12-19. doi:10.21859/jech-03012. [Persian].

11. Cheraghi F, Mortazavi S, Shamsaei F, Moghimbeigi A. Effect of education on management of blood glucose in children with diabetes. Journal of Nursing Education. 2014;3(1):1-11. [Persian].

12. Rajpura JR, Nayak R. Role of illness perceptions and medication beliefs on medication compliance of elderly hypertensive cohorts. J Pharm Pract. 2014;27(1):19-24. doi:10.1177/0897190013493806.

13. Kang CD, Tsang PP, Li WT, et al. Determinants of medication adherence and blood pressure control among hypertensive patients in Hong Kong: a cross-sectional study. Int J Cardiol. 2015;182:250-257. doi:10.1016/j.ijcard.2014.12.064.

14. Saarti S, Hajj A, Karam L, et al. Association between adherence, treatment satisfaction and illness perception in hypertensive patients. J Hum Hypertens. 2016;30(5):341-345. doi:10.1038/ jhh.2015.86.

15. Mohammadi MA, Dadkhah B, Sazavar H, Mozaffari N. The effect of follow up on blood pressure control in hypertensive patients. Journal of Ardabil University of Medical Sciences. 2006;6(2):156162. [Persian].

16. Rahimi A, Ahmadi F, Gholyaf M. Effects of applying continuous care model on quality of life in hemodialysis patients. Razi Journal of Medical Sciences. 2006;13(52):123-34. [Persian].

17. Hacihasanoğlu R, Gözüm S. The effect of patient education and home monitoring on medication compliance, hypertension management, healthy lifestyle behaviours and BMI in a primary health care setting. J Clin Nurs. 2011;20(5-6):692-705. doi:10.1111/j.1365-2702.2010.03534.x.

18. Hashemi S, Tayebi A, Rahimi A, Einolahi B. Examining the effect of continuous care model on adherence to dietary regimen among patients receiving hemodialysis. Iran J Crit Care Nurs. $2015 ; 7(4): 215-20$. 